\title{
Modeling the Movement of Groundwater from the Pits, Surrounded with Tongues of Zhukovsky
}

\author{
Bereslavckii Eduard Naumovich \\ Department of Applied Mathematics and Informatics, University of Civil Aviation, St. Petersburg, Russia
}

Email address:

eduber@mail.ru

\section{To cite this article:}

Bereslavckii Eduard Naumovich. Modeling the Movement of Groundwater from the Pits, Surrounded with Tongues of Zhukovsky. International Journal of Theoretical and Applied Mathematics. Vol. 3, No. 4, 2017, pp. 129-137. doi: 10.11648/j.ijtam.20170304.11

Received: May 8, 2017; Accepted: June 1, 2017; Published: July 21, 2017

\begin{abstract}
In the hydrodynamic statement the filtration low from ditches, walled tongues of Zhukovsky is considered. The fluid moves through the layer of soil, underlain by a well-permeable pressure aquifer, which is contained waterproof area on the roof. For study the infiltration to the free surface of groundwater is formulated a mixed multi-parameter boundary value problem of the theory of analytic function, which is solved by the Polubarinova-Kochina's method and ways the conformal mapping areas of a special kind, which are characteristic for tasks of an underground hydromechanics.
\end{abstract}

Keywords: Filtration, Infiltration, Groundwater Aquifers, Ditch, Tongue of Zhukovsky, Polubarinova-Kochina's Method, Fuchs Differential Equations, Complex Flow Velocity, Conformal Mappings

\section{Introduction}

In the hydrodynamic formulation is considered flat established incompressible fluid filtration by Darcy's law in construction ditches fences tongue Zhukovsky through homogeneous and isotropic soil layer underlained by a wellpermeable pressure aquifer on the roof that provides an impermeable land. During the study infiltration of the free surface groundwater formulated mixed boundary multiparameter problem of analytic function theory, which is solved by the method Polubarinova-Kochina and methods of conformal mappings of a special type, typical of underground fluid mechanics problems. Based on this model, an algorithm of calculation the filtration characteristics in situations when you have to take into account the combined effect of the picture movement of such important factors as the infiltration of the free surface, tight inclusion and backwater from the water well-permeable underlying aquifer. Using the exact analytical dependences and numerical calculations carried out hydrodynamic analysis of the structure and features of the modeled process and the effect of all physical parameters of the circuit on the filtration characteristics. The limiting case of flow associated with the absence of a backwater opaque area or infiltration and degeneration of ditches in a semi-infinite strip on the left of flooding. We give a solution of the problem for the circuit assuming a finite value of flow velocity at the tip of the tongue, which is an analogue of the classical problem of Zhukovsky. The results of calculations for all limiting cases are compared with the main filter model.

The study of filtration flows from the construction pits, fenced symmetrical tongue Zhukovsky, related to work [111]. It was assumed that the water-permeable layer of soil has unlimited power in some cases, in others underlying wellpermeable pressure reservoir was modeled by one or two drains in the form of a horizontal slit Zhukovsky [17]. In some studies examined free filtration, that is, for no backwater, and in some cases - the pressure, the presence of the free surface of neglect. In all these studies, infiltration records are not made. There were used function of Zhukovsky and method of Vedernikov-Pavlovsky, which reduce the case to a conformal mapping of rectilinear polygons and then using the Schwarz-Christoffel formula.

As shown by the practical application of these methods [12-15] their direct use only lead to effective results when the boundary of the movement consists of horizontal and vertical watertight permeable areas. However, in actual hydraulic construction pits (canals, reservoirs) immediately below the overburden, together with the horizontal aquifers higher permeability (pebbles, gravel, coarse sand) often occur also 
waterproof horizontal sections (tight turn, impermeable rock), that fundamentally affects the nature of the filtration processes. In such situations, the use of the SchwarzChristoffel integral does not lead to the goal, as in the areas of integrated flow rate already arise circular polygons, cannot be reduced directly to the straight line.

In contrast to these researches below, as a direct continuation and development of the author's previous results [18-19], we study the problem of the flow of fluid from the pits through the dirt array ultimate power, underlain by a well-permeable pressure aquifer that comprising at its waterproof roof portion of infiltration in the presence of the free surface. We study the most general case of motion in which both permeable areas of the boundary flow filtration takes extreme values and point of zero flow rate enters the tongue. (that apparently has not yet occurred in the literature). There have been extreme cases of course, related to the lack of a backwater, a tight turn or infiltration, and the case of degeneration foundation ditches in a semi-infinite strip on the left of flooding previously studied by V.V. Vedernikov [10]. The results for the circuit that occurs in the absence of critical points in the case where the flow rate at the end of the tongue is finite; the resulting solution is an analogue of the classical problem of Zhukovsky [17].

For the solution of a mixed boundary value multiparameter problem of theory of analytic functions used method Polubarinova-Kochina [12-16] and areas designed for specific species [20-21] of conformal mapping of circular polygons [22-24], which are typical for problems of underground hydromechanics. Accounting for the characteristics of movement allows us to represent the solution through a special, and in some cases, elementary functions, making them easy and convenient use.

Based on the constructed exact analytical dependences and numerical calculations performed by the hydrodynamic analysis of the influence of the physical parameters of scheme on picture effects and highlights some of the features developed models. Mathematical modeling results for all extreme cases compared with the primary filter circuit. Highlights were announced earlier [25].

\section{The Basic Model: Formulation of the Problem}

We consider the planar steady flow from the excavation $A^{\prime} A$ width $2 l$, fenced symmetrical tongue Zhukovsky same length $S$ through the permeable capacity of soil layer $T$ with the underlying aquifer $E^{\prime} E$, containing or underground artesian water wherein the pressure has a constant value $H_{0}$ (Figure 1). On the roof of the reservoir is waterproof section $D^{\prime} D$, simulated horizontal segment of length $2 L$. By the symmetry of pictures motion, we shall restrict the right half of the study filtration area $A B C D E G R$.

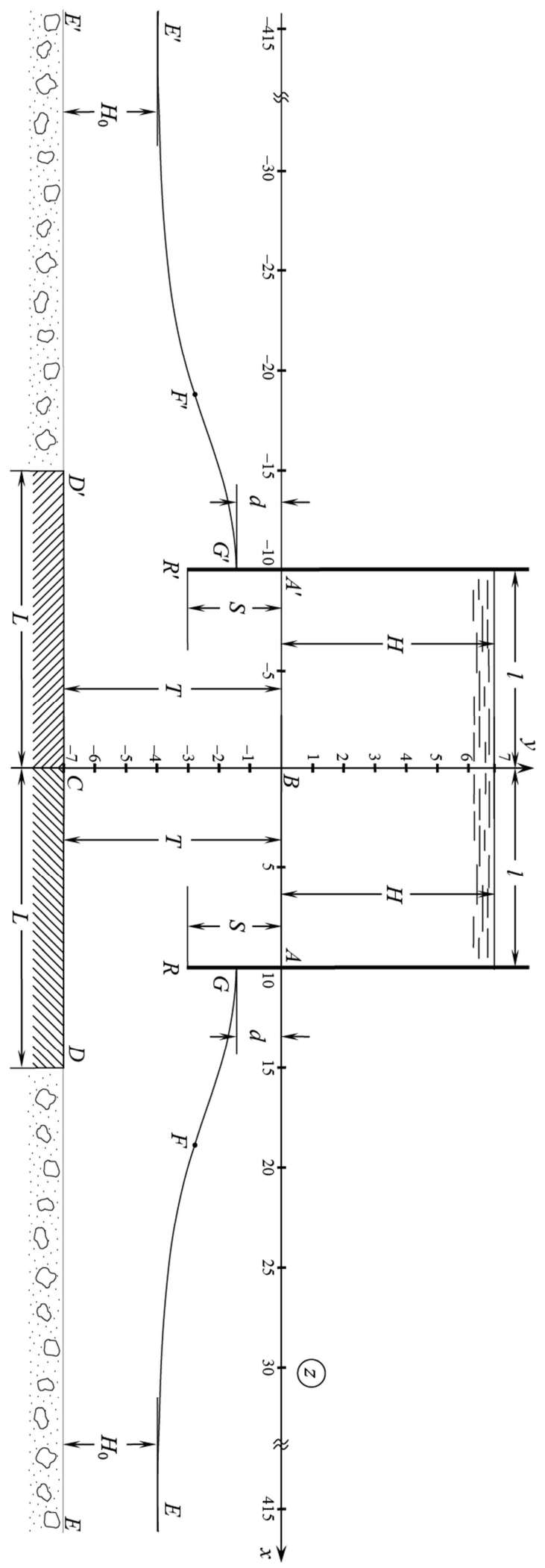

Figure 1. The flow for pattern for base model, calculated in the base case.

Ground water, flowing tongue $A R G$ under the influence of the difference of pressures in the trench and the underlying good permeable aquifer, raised him to a certain height $R G$ and breaking point $M$ zero speed on the tongue, form the free surface of GE, which receives infiltration water intensity $\varepsilon(0$ 
${ }_{\varepsilon}<1$ ), referred to the soil filtration coefficient $\kappa=$ const. The task is to determine the position of the curve $G E$ depression, and, therefore, raise the height of the $\mathrm{RG}$ groundwater of the tongue, that is, the value of $S-d$.

We assume that the movement of groundwater obeys the Darcy's law with a known filtration coefficient $\kappa$ and takes place in a homogeneous and isotropic soil, which is considered incompressible. The rate of flow at the tip of the tongue $v_{R}$ assumed infinite (up to s. 5), the depth of water in the trench $H$ remains unchanged over time.

From a mathematical point of view the problem is to find the complex potential flow $\omega=\varphi+i \psi$, $(\varphi-$ potential speed, $\psi$ - current function) as an analytic function in the field of filtration of the complex coordinates $z=x+i y$ with the following boundary conditions:

$$
\begin{aligned}
& A B: y=0, \varphi=-H ; B C: x=0, \psi=0 ; C D: y=-T, \psi=0 ; A G: x=l, \psi=Q, \\
& D E: y=-T, \varphi=-H_{0} ; G E: \varphi=-y-T, \psi=Q+\varepsilon(x-l),
\end{aligned}
$$

where $Q-$ the desired filtration rate from the pit. A study carried out in terms of the above variables $w$ and $z$, associated with the same name and the actual values $\omega_{f}$ and $z_{f}$ by equalities $\omega=\omega_{f} / \kappa T, z=z_{f} / T$.

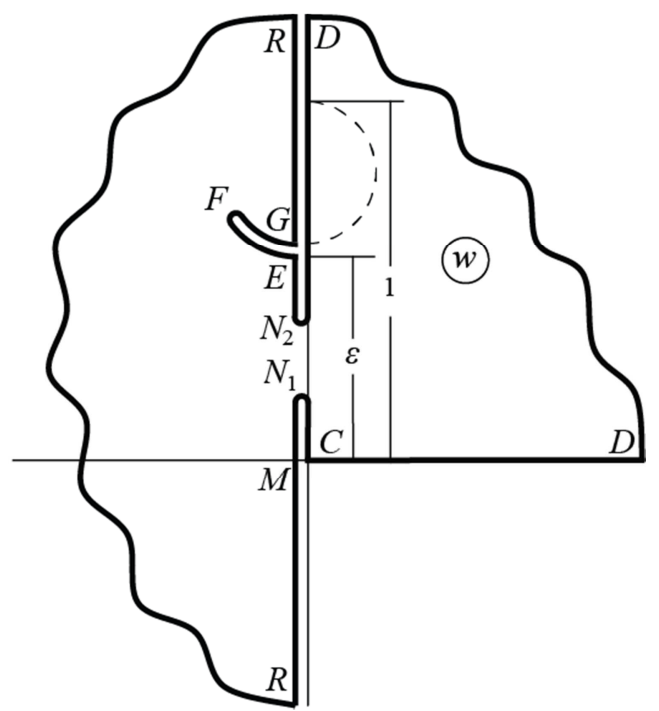

Figure 2. The area of complex velocity $w$.

\section{Construction of the Solution of Boundary Value Problem}

Turning to the field of complex velocity $w$ (Figure 2), corresponding to the boundary conditions (1). This region, which is circular polygon with three cuts, the vertices $N_{l}$ and $N_{2}$ of two of which correspond to the extremes of the stream function at impermeable portions $A B$ and $D E$, belongs to the class of polygons in polar grids [26] and coincides with that in the case previously examined [19] (Figure 2). However, in contrast to [19], in this case, on the border of the movement area, an additional corner point - the point $B$; the total number of singular points is equal to nine, which greatly complicates the task.

To solve the boundary value problem we use the method of Polubarinova-Kochina, which is based on the application of the analytical theory of linear differential equations of the Fuchs class [12, 16, 27]. We introduce the auxiliary parametric variable $\zeta$ and function $z(\zeta)$, conformally maps the upper half in the region $z$ accordance with points

$$
\zeta_{E}=0, \zeta_{G}=1, \zeta_{C}=k^{-2}, \zeta_{D}=\infty
$$

and also derivatives

$$
\Omega=\frac{d \omega}{d \zeta}, Z=\frac{d z}{d \zeta} .
$$

Defining characteristic indicators of functions $\Omega$ and $Z$ near the regular singular points [12], we find that they are linear combinations of the two branches next Riemann function $[12,27]$ :

$$
\begin{aligned}
& P\left\{\begin{array}{cccccccccc}
0 & \zeta_{F} & 1 & \zeta_{A} & \zeta_{N_{1}} & \zeta_{B} & k^{-2} & \zeta_{N_{2}} & \infty & \\
-1 & 0 & -1 / 2 & -1 / 2 & 0 & -1 / 2 & -1 / 2 & 0 & 3 / 2 & \zeta \\
-1 / 2 & 2 & 0 & -1 / 2 & 2 & -1 / 2 & 0 & 2 & 2 &
\end{array}\right\}= \\
& =\frac{Y}{\zeta \sqrt{(1-\zeta)\left(\zeta_{A}-\zeta\right)\left(\zeta_{B}-\zeta\right)\left(1-k^{2} \zeta\right)}}, \\
& Y=P\left\{\begin{array}{cccccccc}
0 & \zeta_{F} & 1 & \zeta_{N_{1}} & k^{-2} & \zeta_{N_{2}} & \infty & \\
0 & 0 & 0 & 0 & 0 & 0 & -3 / 2 & \zeta \\
1 / 2 & 2 & 1 / 2 & 2 & 1 / 2 & 2 & -1 &
\end{array}\right\} .
\end{aligned}
$$

It is evident that the point $\zeta=\zeta_{A}$ and $\zeta=\zeta_{B}$ - ordinary point of function $Y$, which represents the last symbol of Riemann. This symbol of Riemann corresponds to a linear differential equation of Fuchs class with seven regular singular points, which 
is quite typical for the problems of underground hydromechanics $[20,21]$ and in this case has the form

$$
\begin{aligned}
& Y^{\prime \prime}+\left[\frac{1}{2}\left(\frac{1}{\zeta}+\frac{1}{\zeta-1}+\frac{1}{\zeta-k^{-2}}\right)-\left(\frac{1}{\zeta-\zeta_{F}}+\frac{1}{\zeta-\zeta_{N_{1}}}+\frac{1}{\zeta-\zeta_{N_{2}}}\right)\right] Y^{\prime}+ \\
& +\frac{3 \zeta^{3}+\lambda_{2} \zeta^{2}+\lambda_{1} \zeta+\lambda_{0}}{\zeta(\zeta-1)\left(\zeta-k^{-2}\right)\left(\zeta-\zeta_{F}\right)\left(\zeta-\zeta_{N_{1}}\right)\left(\zeta-\zeta_{N_{2}}\right)} Y=0 .
\end{aligned}
$$

Recall that in addition to affix $\zeta_{F}, \zeta_{N 1}$ and $\zeta_{N 2}$ in equation (4) accessory parameters $\lambda_{0}, \lambda_{1}$ and $\lambda_{2}$ are unknown at the statement of the problem and should be identified during its solution.

Change of variables

$$
\zeta=\operatorname{sn}^{2}(2 K \tau, k)
$$

transforms the upper half into a rectangle $\zeta \tau$ plane:

$0<\operatorname{Re} \tau<1 / 2,0<\operatorname{Im} \tau<\rho / 2, \rho(k)=K^{\prime} / K, K^{\prime}=K\left(k^{\prime}\right), k^{\prime}=\sqrt{1-k^{2}}$, where $K(k)-$ complete elliptic integral of the first kind for $k$ module $[27,28]$ at the corresponding points

$$
\tau \mathrm{E}=0, \tau \mathrm{G}=1 / 2, \tau \mathrm{C}=(1+i \rho) / 2, \tau \mathrm{D}=i \rho / 2,
$$

and the integrals $Y$ of equation (4), which correspond to the symbol of Riemann (3) and constructed according to the method developed earlier [22-24], converts to the form:

$$
Y_{1,2}(\tau)=\vartheta_{0}^{-3}(\tau) \vartheta_{1}(\tau \pm i \gamma) \vartheta_{2}(\tau \pm i \alpha) \vartheta_{2}(\tau \mp i \beta) \exp ( \pm i \pi \tau) .
$$

Here $s n(u, k)$ - Jacobi elliptic function (sinus) for k module, $\vartheta_{0}(\tau), \vartheta_{1}(\tau)$ и $\vartheta_{2}(\tau)$ - theta function with parameter q $=\exp (-$ $\pi \rho)$, which It is uniquely associated with the module $\mathrm{k}[27,28], \alpha, \beta, \gamma-$ some suitable constants.

Taking into account the relations (3), (5) and (6), as well as the fact that the function $w=d \omega / d z$ has previous form [18, 25]

$$
\begin{gathered}
w=i \sqrt{\varepsilon} \frac{\chi^{+}(\tau)}{\chi^{-}(\tau)}, \chi^{ \pm}(\tau)=(1+\sqrt{\varepsilon}) Y_{1}(\tau) \pm(1-\sqrt{\varepsilon}) Y_{2}(\tau), \\
\sqrt{\varepsilon}=\operatorname{th} \pi(\rho / 2+\beta-\alpha-\gamma),
\end{gathered}
$$

we arrive at the source dependencies

$$
\begin{aligned}
& \Omega=-\sqrt{\varepsilon} N \frac{\chi^{+}(\tau)}{\Delta(\tau)}, Z=i N \frac{\chi^{-}(\tau)}{\Delta(\tau)} \\
& \Delta(\tau)=\operatorname{sn}(2 K \tau, k) \sqrt{\left[1-\left(1-k^{\prime 2} A^{2}\right) \operatorname{sn}^{2}(2 K \tau, k)\right]\left[1-\left(1-k^{\prime 2} B^{2}\right) \operatorname{sn}^{2}(2 K \tau, k)\right]} .
\end{aligned}
$$

Here $N>0$ - constant scale simulation, $A=\operatorname{sn}\left(2 K a, k^{\prime}\right), B$ $=\operatorname{sn}\left(2 K b, k^{\prime}\right), a$ and $b-$ unknown ordinates of points $A$ and $B$ domain $\tau$. The representations (9) constant conformal mapping $\alpha, \beta$ and $\gamma$, which are connected by the relation (8), subject to the conditions

$$
0<\alpha<r<\beta<m<a<b<\rho / 2,0<\gamma<\rho / 2,
$$

regulating the position in the current boundary field points of zero velocity $M$ and the tip of the tongue $R$, and the well $N_{l}$ and $N_{2} ; m$ and $r$ - unknown ordinates of the points $M$ and $R$ in the plane $\tau$.

You can verify that the functions (9) satisfy the conditions (1), reformulated in terms of functions $d \omega / d \tau$ and $d z / d \tau$, and thus, are parametric solution of the original boundary value problem.

Writing equations (9) for different parts of the border region $\tau$ followed by integration over the whole contour of the sub-area leads to the closure motion field and thus serves as a computation control.

The result is an expression for defined and the desired geometrical and filtration properties of the model

$$
\begin{gathered}
\int_{r}^{a} Y_{R A} d t=S, \int_{a}^{b} X_{A B} d t=l, \int_{0}^{1 / 2} X_{C D} d t=L, \int_{b}^{\rho / 2} Y_{B C} d t=T, \\
\int_{b}^{\rho / 2} \Phi_{B C} d t-\int_{0}^{1 / 2} \Phi_{C D} d t=H-H_{0}, \\
d=T-H_{0}-\int_{0}^{1 / 2} \Phi_{E G} d t, Q=\int_{a}^{b} \Psi_{B C} d t
\end{gathered}
$$

and coordinate $E G$ depression curve points:

$$
x_{E G}(u)=l+\int_{u}^{1 / 2} X_{E G} d t, y_{E G}(u)=-d+\int_{u}^{1 / 2} Y_{E G} d t, 0 \leq u \leq 1 / 2 .
$$


Control accounts are other expressions for $d, L$, and filtration flow rate $Q$

$$
\begin{gathered}
d=T-H_{0}-\int_{0}^{1 / 2} Y_{E G} d t, L=l-\int_{0}^{1 / 2} X_{E G} d t-\int_{0}^{\rho / 2} X_{D E} d t \\
Q=\int_{0}^{\rho / 2} \Psi_{D E} d t-\varepsilon \int_{0}^{1 / 2} X_{E G} d t
\end{gathered}
$$

In the formulas $(11)-(14)$ integrands - expression right side of (9) in the respective sections of the circuit area $T$.

\section{The Numerical Results for the Main Filter Model: Discussion of Results}

The representations (9) - (14) contain seven unknown constants: ordinates of $a, b, r$ inverse images of points $A, B, R$ in the plane $T$, the parameters of conformal mapping of $\alpha, \beta$, $\gamma$, satisfying (8) and the inequalities (10), as well as a module the $k(0<k<1)$ and constant modeling $N$. To determine them at specified $S, l, L, H$, and $T$ is the set of equations (11), which are used together with the relations
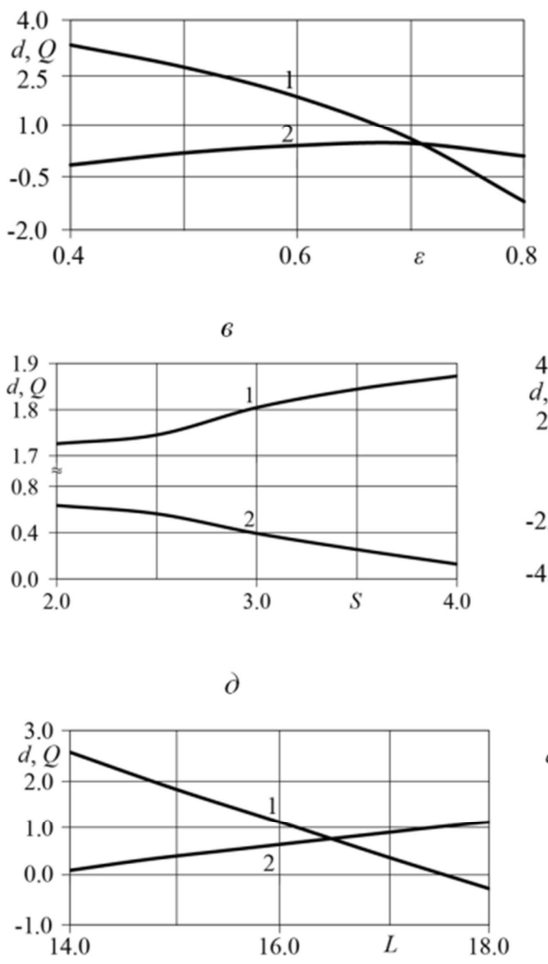

$$
\begin{gathered}
w^{-1}(1 / 2+i r)=0 \\
\int_{0}^{1 / 2}\left(\Phi_{E G}+\Phi_{C D}\right) d t+\int_{0}^{a} \Phi_{G A} d t+\int_{b}^{\rho / 2} \Phi_{B C} d t=0 .
\end{gathered}
$$

The first of these relations means that the rate at the end of the tongue tends to infinity, and the second follows directly from consideration of the boundary conditions (1). After determining the unknown constants are unknown quantities $d$ and $Q$ from the formulas (12) and, finally, by the formulas (13) calculated coordinates of the free surface $E G$ points.

Figure 1 shows a flow pattern, calculated at

$$
\varepsilon=0.6, T=7, S=3, H_{0}=3, L=15, H=7, l=10
$$

(Baseline values). Table 1 and 2 (varies within the acceptable range of one of these parameters, and the rest are recorded baseline values) shows the results of calculations of the effect of defining the physical parameters of $\varepsilon, T, S, H_{0}, L$, $H$ and $l$ at depth $d$ ( $d$ negative values indicate that the free surface is raised above the abscissa) and consumption $Q$. Figure 3 shows depending on the value of $d$ (curve 1) and filtration flow rate $Q$ (curve 2) of $\varepsilon, T, S, H_{0}, L, H, l$.

б
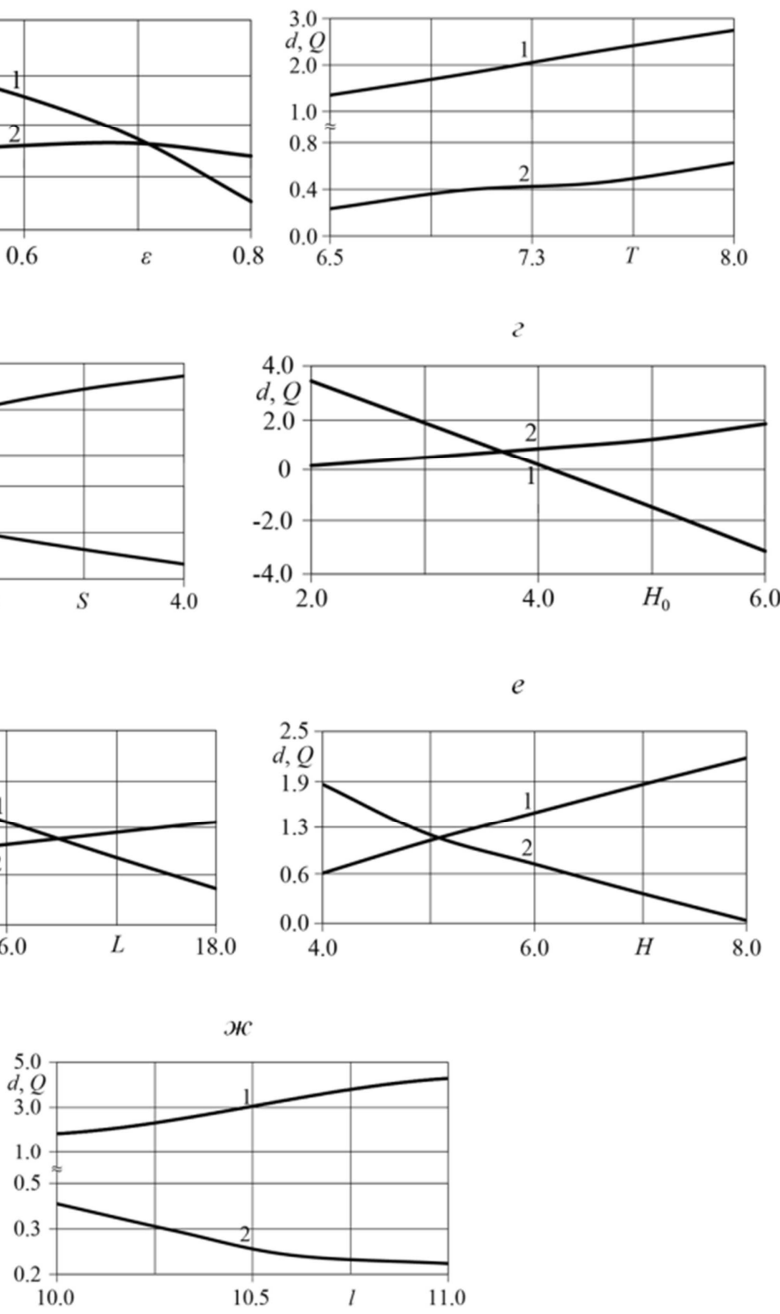

Figure 3. The dependence of $d$ (curves 1 ) and $Q$ (curves 2 ) for the base model from $\mathcal{E}, T, S, H_{0}, L, H, l$. 
Analysis of the data tables and charts to the following conclusions.

First of all, attention is drawn to the same qualitative character of dependent variables $d$ and $Q$ on the parameters $T$ and $l, S$ and $H$, and at the same time the opposite of the behavior desired characteristics when changing the parameters $S$ and $H$ on one side and on the other $-L$ and $H_{0}$.

Increased infiltration intensity, width tight turn and head in the underlying reservoir and power reduction layer of the tongue length, water pressure in the pit and its width lead to reduction of the depth $d$, which increase ordinate of point $G$ yield curve depression from under the tongue.

Thus, according to the Table 1 and 2 , an increase in the parameters $\varepsilon, S, H_{0}$ and $l$ in the 1.6, 2.0, 2.0 and 1.1 times accompanies the change in the value of $d$ in $2.2,1.1,1.3$ and 1.6 times, respectively. However, the greatest impact on the depth $d$ has an impermeable area: Data Tables 2 show that increasing the width $L$ of $28 \%$ total depth $d$ increases almost 10 -fold.

Table 1. The results of calculations of values of $d$ and $Q$ for the base model when variation $\mathcal{E}, T, S, H_{0 .}$.

\begin{tabular}{|c|c|c|c|c|c|c|c|c|c|c|c|}
\hline$\varepsilon$ & d & $\mathbf{Q}$ & $\mathbf{T}$ & d & $\mathbf{Q}$ & $\mathbf{S}$ & d & $\mathbf{Q}$ & $\mathbf{H}_{0}$ & d & $\mathbf{Q}$ \\
\hline 0.5 & 2.651 & 0.182 & 6.5 & 1.349 & 0.234 & 2.0 & 1.726 & 0.635 & 2.0 & 3.155 & 0.038 \\
\hline 0.6 & 1.804 & 0.394 & 7.0 & 1.804 & 0.394 & 2.5 & 1.745 & 0563 & 4.0 & 0.441 & 0.769 \\
\hline 0.7 & 0.586 & 0.457 & 7.5 & 2.299 & 0.457 & 3.5 & 1.844 & 0255 & 5.0 & -0.93 & 1.159 \\
\hline 0.8 & -1.195 & 0.094 & 8.0 & 2.745 & 0.627 & 4.0 & 1.873 & 0.129 & 6.0 & -2.35 & 1.815 \\
\hline
\end{tabular}

Table 2. The results of calculations of values of $d$ and $Q$ for the base model when variation $L, H, l$.

\begin{tabular}{lllllllll}
\hline $\mathbf{L}$ & $\mathbf{d}$ & $\mathbf{Q}$ & $\mathbf{H}$ & $\mathbf{d}$ & $\mathbf{Q}$ & $\mathbf{l}$ & $\mathbf{d}$ & $\mathbf{Q}$ \\
\hline 14 & 2.555 & 0.086 & 4.0 & 0.650 & 1.815 & 10.0 & 1.804 & 0.394 \\
16 & 1116 & 0.599 & 5.0 & 1.070 & 1.159 & 10.3 & 2.018 & 0.320 \\
17 & 0.413 & 0.888 & 6.0 & 1.441 & 0.769 & 10.6 & 2.229 & 0.253 \\
18 & -0.256 & 1.084 & 8.0 & 2.155 & 0.038 & 11.0 & 2.478 & 0.230 \\
\hline
\end{tabular}

When $\varepsilon=0.8, H_{0}=5$ and 6 and $L=18$, that is, for sufficiently large values of the parameters $e, H_{0}$ and $L$ free surface rises above the $\mathrm{x}$-axis, the value of $d$ becomes negative. If we introduce the dimensionless quantity $h(d)=$ $(S-d) / S, h(S)=0$, which characterizes the relative height of the lift groundwater of the tongue, the values for these parameters $\varepsilon$, and $L, H_{0}$, we get $h(-1.1945)=1.3981, h(-$ $0.9297)=1.3099, h(-2.3500)=1.7833$ and $h(-0.2560)=$ 1.0853 respectively, and the value $h$ increases with increasing ordinate of point $G$ yield curve depression from under the tongue.

Dependencies depth $d$ on the parameters $T, H_{0}, L$ and $H$ are close to linear.

As for consumption, the tight turn with increasing width of the $Q$-value is also increasing: Table 2 shows that an increase in the parameter $L$ to 1.28 times the flow rate implies an increase of more than 12 times. Thus, it revealed a significant propping up effect of impervious area in relation to the filtering of the pit.

This flow behavior is clearly observed how with increasing layer thickness, the width of the pit, and the pressure in the underlying horizon, and with a decrease in the length of the tongue and head to tail water. Table 2 shows that reduction of the $H$ only 2 times accompanied by an increase in flow rate $Q$ is almost 48 times, indicating that the greatest impact on the consumption of water pressure in the pit.

\section{Limiting Cases}

$1^{0}$. Case $H_{0}=0$.

Let us consider, first of all, on the absence of pressurization, i.e. moving when $H_{0}=0$. The solution for this limiting case is obtained from relations (9) - (14) with $\gamma=\gamma_{*}=0$. With this value of the parameter $\gamma$ circular incision EG field $\mathrm{w}$, transforming, degenerates in the right semicircle (dashed line in Figure 2) and thus the source area is transformed into a circular hexagon, which falls right side of the semicircle $|w-i(1+\varepsilon) / 2|<(1-\varepsilon) / 2$. In the course of the plane $z$ for $\gamma=\gamma_{*}$ depression curve flattens out at point $E$, deviates from that merges its inflection point $F$ and exits to the roof of the underlying horizon at right angle.

Figure 4 shows the flow pattern is calculated with the basic version

$$
\varepsilon=0.6, T=7, S=3, L=15, H=5, l=10 .
$$

Table 3 and 4 show the results of numerical calculations of the impact of the physical parameters of $\varepsilon, T, S, L, H$ and $l$ on the filtration characteristics $d$ and $Q$.

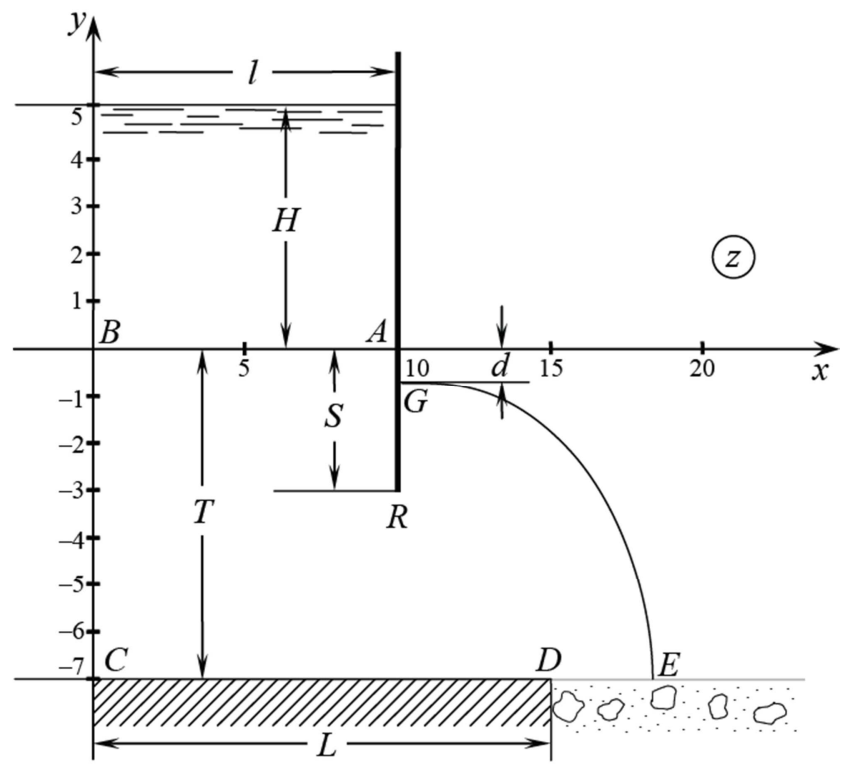

Figure 4. The flow pattern at $H_{0}=0$, calculated in the base case $\varepsilon=0.6, T=7$, $S=3, L=15, H=5, l=10$. 
Table 3. The results of calculations of values of $d$ and $Q$ for the case $H_{0}=0$ when variation $\mathcal{E}, T, S$.

\begin{tabular}{lllllllll}
\hline $\boldsymbol{\varepsilon}$ & $\mathbf{d}$ & $\mathbf{Q}$ & $\mathbf{T}$ & $\mathbf{d}$ & $\mathbf{Q}$ & $\mathbf{S}$ & $\mathbf{d}$ & $\mathbf{Q}$ \\
\hline 0.4 & 1.974 & 0.624 & 6.0 & -0.606 & 0.344 & 2.0 & 1.066 & 0.179 \\
0.5 & 1.395 & 0.448 & 6.5 & -0.042 & 0.315 & 2.5 & 0.747 & 0.232 \\
0.6 & 0.572 & 0.276 & 7.5 & 1.258 & 0.226 & 3.5 & 0.471 & 0.307 \\
0.7 & -0.657 & 0.107 & 8.0 & 2.085 & 0.172 & 4.0 & 0.419 & 0.327 \\
\hline
\end{tabular}

A comparison with the results for the base model shows that there is a completely different behavior of the values of $d$ and $Q$ by varying the parameters of $S$ and $H$ : now with increasing length of the dowel head and tail-to the contrary, $d$ depth decreases and the flow rate $Q$ increases. Moreover, the behavior of the quantities $Q$ and $d$, parameters $\varepsilon$ and $L$ becomes qualitatively similar, and the parameters $\varepsilon$ and $l-$ opposite.

Just as in the main filter circuit, a considerable influence on the value $d$ has a water pressure in pond: Table 4 shows that change the argument $H$ on $50 \%$ corresponds to a decrease of depth $\mathrm{d}$ almost 8 times. The greatest impact, as before, has a tight inclusion. Table 4 also shows that change the width $L$ of just 1.3 times the flow rate implies a reduction by more than 18 times.

Table 4. The results of calculations of values of $d$ and $Q$ for the case $H_{0}=0$ when variation $L, H, l$.

\begin{tabular}{lllllllll}
\hline $\mathbf{L}$ & $\mathbf{d}$ & $\mathbf{Q}$ & $\mathbf{H}$ & $\mathbf{d}$ & $\mathbf{Q}$ & $\mathbf{l}$ & $\mathbf{d}$ & $\mathbf{Q}$ \\
\hline 12 & 2.506 & 1.425 & 4.5 & 1.013 & 0.041 & 9.50 & 0.223 & 0.108 \\
13 & 1.902 & 1.009 & 5.0 & 0.571 & 0.276 & 10.0 & 0.571 & 0.276 \\
14 & 1.252 & 0.628 & 5.5 & 10.174 & 0.513 & 10.5 & 0.914 & 0.449 \\
15 & 0.571 & 0.276 & 6.0 & -0.191 & 0.753 & 11.0 & 1.248 & 0.630 \\
\hline
\end{tabular}

Impermeable portion most significant impact also on the flow rate by changing the last 26.6 times.

For values of $\varepsilon=0.7, \mathrm{~T}=6$ and $6.5, L=18, H=6$ and $l=$ 9 , in which the value of $d$ becomes non-positive, the parameter $h$ takes values $h=1.2191,1.2020,1.0140,1.0443$, 1.0638 and 1.0442 , respectively.

\section{$2^{0}$. Case $l=\infty$.}

Consider the case where the width of the pit increases indefinitely. If you make the transformation $z{ }^{\prime}=z+l$, moving the point $A^{\prime}$ to the origin and fix all the physical parameters of the model then with increasing width of the pit $l$, conformal mapping constant $b \rightarrow b_{*}=\rho / 2$. In the limit of $l=\infty$ parameters are $b=b_{*}, B=1$. In the $z$ plane of motion points $B$ and $C$ merge to infinity, so the filtration area becomes a semiinfinite strip on the left of flooding. In (9)-(14) where $b=b$ * flow results obtained previously $[18,25]$.

$$
3^{0} \text {. Case } L=0 \text {. }
$$

In the main filter scheme discussed above, the absence of such a powerful factors as the tight incorporation, being limiting, serves as a background in the evaluation of the role of infiltration and pressure in the underlying reservoir and allows more contrast to trace the interaction of important physical parameters, such as $\varepsilon$ and $H_{0}$.

If there is no impervious area on the roof of the underlying aquifer, the latter along its entire length becomes wellpermeable. When you merge points $C, D$ in the area of complex velocity her left half-plane w cut off, a circular incision $E G$ moves in the right half-plane, and source area is transformed into a circular triangle (Figure 5). In the movement plane $z$ point $D$, merging with point $C$, yield on the axis, and rectangle of the plane $\tau$ is transformed to halfstrip $0<\operatorname{Re} \tau<1 / 2,0<\operatorname{Im} \tau<\infty$, because parameter $\rho=K^{\prime} / K=$ $\infty, K=\pi / 2$.

The solution for this limiting case is obtained from the formulas (9)-(14), if we put $k=0$ in these formulas and consider that in this case the elliptic functions degenerate into trigonometric and theta-functions are cut off in their first terms or constants:

$$
\begin{aligned}
& \Omega=-\varepsilon N \frac{\sin 2 r^{\prime} \sin 2\left(\tau-m^{\prime}\right)}{\sin 2 m^{\prime} \cos \tau \Delta(\tau)}, Z=i N \frac{\sin 2\left(\tau-r^{\prime}\right)}{\cos \tau \Delta(\tau)}, \\
& \Delta(\tau)=\sqrt{\left(a-\sin ^{2} \tau\right)\left(b-\sin ^{2} \tau\right)\left(c-\sin ^{2} \tau\right)}, \quad \varepsilon=\operatorname{tg} 2 m^{\prime} \operatorname{ctg} 2 r^{\prime} .
\end{aligned}
$$

Here $m^{\prime}=\arcsin \sqrt{m}, r^{\prime}=\arcsin \sqrt{r}, m^{\prime}, r^{\prime}, c^{\prime}\left(0<m^{\prime}<r^{\prime}\right.$ $\left.<a^{\prime}<b^{\prime}<c^{\prime}<\pi / 2\right)$ are inverse images of points $M, R, C$ on the horizontal axis of the plane $\tau$. This case is described in detail previously [19], where an analysis of the influence all of the physical parameters of the model.

$4^{0}$. Case $\varepsilon=0$.

Within the case $L=0$ we focus on the absence of infiltration. Given the connection between the parameters $m^{\prime}$ and $r^{\prime}$, noted in (16), we see that in the case where $\varepsilon=0$, there is implying the solution of the problem of dependences (16) where $m^{\prime}=0$ that is when the plane $\tau$ point depression curve $G, E$ are merged at the origin with point M zero speed. There is a solution to the problem, first examined V. V. Vedernikov [10], only in the other way.

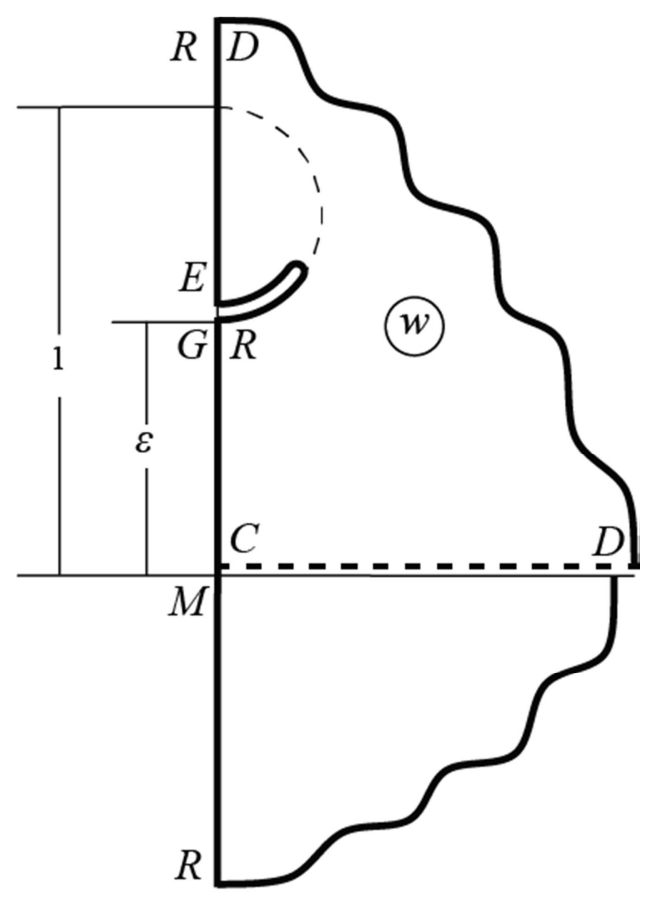

Figure 5. The area of complex velocity at $L=0$. 


\section{The Case of a Finite Value of Flow Rate at the End of the Dowel: Analysis of Zhukovsky Problem}

Within the boundary value problem (1) we consider the case when the flow speed on the end of a tongue $v_{R}, 0<v_{R}<$ $\varepsilon$ finite and stream function in the permeable sections $A B$ and $D E$ has no extremes. Then, in the complex velocity $w$ there are disappearing both of the vertical incisions, the left halfplane is truncated, as previously in the case of $L=0$, but, unlike the latter, $M R$ portion is transferred to the first quadrant (dashed line in Figure 5). As a result, the source region is transformed into a circular pentagon. Parametric solution of the problem is formally the same form (9) with the replacement of the integrals $Y_{1,2}(\tau)$ and regular conformal mapping $\alpha$ and $\beta$ on next $[29,30]$ :

$$
\begin{gathered}
Y_{1,2}(\tau)=\vartheta_{0}^{-1}(\tau) \vartheta_{1}(\tau \pm i \gamma) \exp ( \pm i \pi \tau), \\
\alpha=\beta=(1+i \rho) / 2
\end{gathered}
$$

A similar solution to the problem in the case of lack of backwater flows from ideas (9), (17), (18) when $\gamma=\gamma_{*}$.

The analysis of the numerical results shows that in the case of $v_{R}<\infty$ retained the qualitative nature of the dependencies of the filtration rate of the physical parameters of the circuit typical case when $v_{R}<\infty$. For example, there is the same as before, the flow behavior of $T$ and $l$ values from one side and the opposite character of $S$ and $H$ parameters - on the other. Significant impact on consumption on $Q$, and as before, have infiltration, a dense layer switch and power.

Figure 6 shows the pattern of motion calculated at

$$
\varepsilon=0.5, T=6, S=3, H_{0}=0, L=16.2, H=3, l=15 .
$$

Noteworthy is the fact that all settlement options is $d=S$, and therefore, the value of $h(d)=h(S)=0$. This means that in the plane of the current point $G$ yield curve depression out of the tongue merges with the $R$ point of his sharp; from the review of the field, comprehensive rate $w$ implies that in this case the speed at the end of the tongue is equal to the infiltration rate: $v_{R}=\varepsilon, 0<\varepsilon<1$.

If you make the transformation $\tau^{\prime}=1 / 2+i \rho^{\prime} \tau$, sending rectangle auxiliary variable $\tau$ in the like with parameter $\rho^{\prime}=$ $1 / \rho=K / K^{\prime}$, then the corresponding primary filter circuit on the parameters of inequality (10) takes the form:

$$
0<b^{\prime}<a^{\prime}<r^{\prime}<1 / 2
$$

where $b^{\prime}, a^{\prime}, r^{\prime}$ - abscissa's inverse images of points $B, A, R$ in the plane $\tau$.

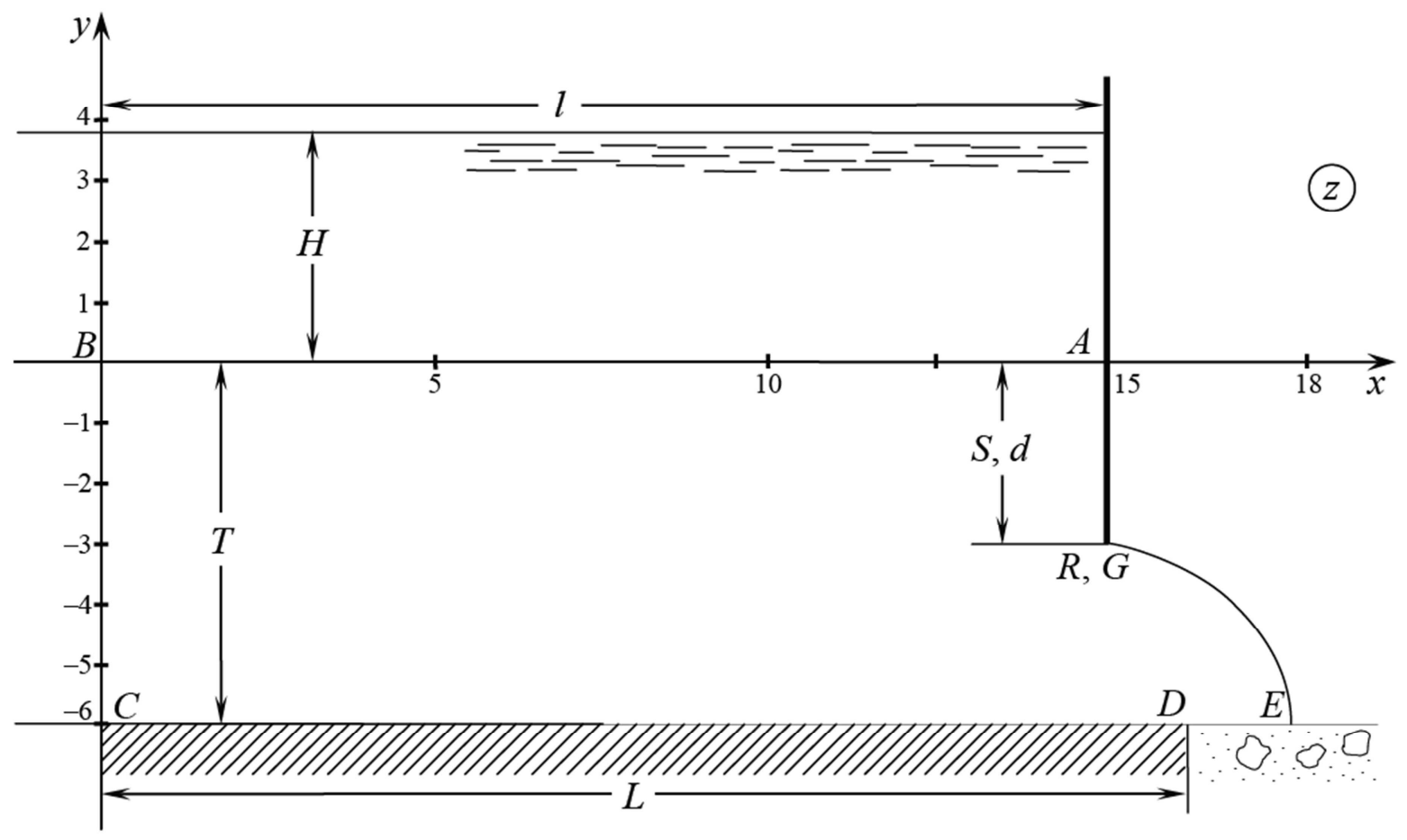

Figure 6. The flow pattern at $0<v_{R}<\varepsilon$ in base case $\varepsilon=0.5, T=6, S=3, H_{0}=0, L=16,2, H=3, l=15$.

Calculations show that for any value of the intensity of infiltration $\varepsilon(0<\varepsilon<1)$ the ratio of $d=S$ holds only for single values of $r^{\prime}$ - its limit $r_{*}^{\prime}$, when the plane $\tau^{\prime}$ merge point $G$, and $R: r^{\prime}=r_{*}^{\prime}=1 / 2$. All other valid values lead to inconsistencies with the real picture of the flow - the relationship $d>S$, i.e., the separation of the flow. A similar result in the limit for this model when the water permeable layer of soil has unlimited power, there is no impenetrable plot and infiltration, when $T=\infty\left(k^{\prime}=0, k=1\right), L=0$ $\left(b^{\prime}=b_{*}^{\prime}=0\right)$ и $\varepsilon=0\left(m^{\prime}=0\right)$, was first obtained in due time, N. E. Zhukovsky [17]. The solution for this limiting case is obtained from dependencies (9), (17), (18), if you put in them $K=\infty, K^{\prime}=\pi / 2, k^{\prime}=0, k=1, b^{\prime}=0, q^{\prime}=0$ and consider that in this case the elliptic functions degenerate into hyperbolic, 
and theta-functions which, this time characterized by the parameter $\mathrm{q}^{\prime}=0$, break off on their first terms or constants. Thus, in the limiting case study scheme Zhukovskogo obtained solution of the problem only by other means.

\section{Conclusion}

Executed in consideration of flows of pits transformed from the basic filter circuits may serve to illustrate the variety of physical content multiparametric boundary value problem with a free surface. An important place is occupied with the extreme cases that seem to be bordered by the original simulated process in describing its boundary value problem and lead to transformations considered the main filter circuit. Access to such extreme cases is carried out on reaching any of the unknown parameters of a conformal mapping of its critical values.

\section{References}

[1] V. V. Vedernikov. Influence of capillary rise in the filtration channel // Hydraulic engineering. 1935. №5. P. 20-27.

[2] V. V. Vedernikov. Influence of soil capillary seepage with free surface // Dokl. USSR Academy of Sciences. 1936. T. III (12). № 4 (99). P.157-161.

[3] V. I. Aravin. The inflow of groundwater to the trenches, fences tongue // Math. VNIIG. 1937. T. 20. S.74-89.

[4] V. S. Kozlov. Hydromechanical calculation sheet jumpers // Math. USSR Academy of Sciences. OTN. 1939. №6. P.89110 .

[5] V. V. Vedernikov. Filtration Theory and its application in the field of irrigation and drainage. M.; L.: Gosstroiizdat. 1939. $248 \mathrm{p}$.

[6] F. B. Nelson-Skornyakov. Hydro water flow calculation to the trench // Math. USSR Academy of Sciences. OTN. 1943. №7. P. 90-94.

[7] F. B. Nelson-Skornyakov. Some cases pritekaniya ground water from the river to the career (trench) // Math. USSR Academy of Sciences. OTN. 1944. №3. S.209-220.

[8] F. B. Nelson-Skornyakov. Filtration in a homogeneous medium. M.: Soviet science. 1947. 279 p.; 2nd ed. 1949. 568 p.

[9] V. I. Aravin and S. N. Numerov. Filtration calculations of hydraulic structures. M.: Stroyizdat. 1948. 227 p.

[10] V. V. Vedernikov. Filtering in the presence of drainage or aquifer // Dokl. USSR Academy of Sciences. 1949. T.69. №5. S.619-622.

[11] L. D Aptekar. Questions filtration calculation of horizontal drainage shipping locks and dry docks // Izv. VNIIG. 1951. T.46. S.80-105.

[12] P. Y. Polubarinova-Kochina Theory of movement of groundwater. M.: Gostekhizdat. 1952. 676 p.; 2nd ed. M.: Nauka. 1977. 664 p.
[13] V. I. Aravin and S. N. Numerov. Theory fluids movement deformable porous media. M.: Gostekhizdat. 1953. 616 p.

[14] Development of filtration theory research in the USSR (19171967). M.: Nauka. 1969. 545 p.

[15] G. K. Mikhailov, V. N. Nicholaevskii. The movement of fluids in porous media // Mechanics in the USSR for 50 years. M.: Nauka. 1970. Vol. 2. S. 585-648.

[16] P. Y. Polubarinova-Kochina. Selected tr. The hydrodynamics and the theory of filtration. M.: Nauka. 1991.351 p.

[17] N. E. Zhukovsky. Seepage of water through the dam // Coll. Vol. M.: Gostekhizdat. 1950. T.7. S.297-332.

[18] E. N. Bereslavskii. Some hydrodynamic models related to the problem of the flow of Zhukovsky tongue // Dokl. Russian Academy of Sciences. 2013. T.448. Number 5. S.529-533.

[19] E. N. Bereslavskii. On some mathematical models related to the problem of the flow of Zhukovsky tongue // J. Appl. Math. and Mech. 2014. T.78. Vol.3. Pp 394-410.

[20] E. N. Bereslavskii, P. Y. Polubarinova-Kochina. On some classes of equations of Fuchs in hydro and Aeromechanics // Math. Russian Academy of Sciences. Fluid Dynamics. 1992. №5. P.3-7.

[21] E. N. Bereslavskii, P. Y. Polubarinova-Kochina. Differential equations of Fuchs class encountered some problems of mechanics of liquids and gases // Math. Russian Academy of Sciences. Fluid Dynamics. 1997. № 5. P. 9-17.

[22] E. N. Bereslavskii. Conformal mapping of some circular polygons on a rectangle // Izv. Vuzov. Mathematics. 1980. №5. P.3-7.

[23] E. N. Bereslavskii. Differential equations of Fuchs class associated with the conformal mapping of circular polygons in polar grids // Differents. equation. 1997. T.33. No. 3. P.296301 .

[24] E. N. Bereslavskii. On some differential equations class Fuchs, occurring in problems of mechanics of liquids and gases // Differents. equation. 2012. T.48. №4. P.590-594.

[25] E. N. Bereslavskii. Application of Polubarinova-Kochina to study seepage flows from the trenches, fenced tongue Zhukovsky // Dokl. RAN.2014. T.455. Number 6. P. 651-655.

[26] Koppenfels W, Stallmann F. Praxis der Konformen Abbildung. Berlin: Springer, $1959=$ Koppenfels V., F. Shtalman practice of conformal mappings. M.: IL. 1963.406 p.

[27] V. V. Golubev. Lectures on the analytic theory of differential equations. M.; L.: Gostekhizdat. 1950. 436 p.

[28] I. S. Gradshtein, I. M. Ryzhik. Tables of integrals, sums, series and products. M.: Nauka. 1971. 1108 p.

[29] E. N. Bereslavskii. Simulation flow tongue Zhukovsky // Reports of the Russian Academy of Sciences. 2011. T.440. №1. S.47-51.

[30] E. N. Bereslavskii. About groundwater flow regime at the tongue Zhukovsky // J. Appl.Math. and Mech. 2011. T.75. Vol. 2. P.303-313. 